\title{
Special structure, decentralization and symmetry for linear systems
}

Michiel Hazewinkel

Centre for Mathematics and Computer Science

PO Box 4079

1009 AB Amsterdam
Clyde F. Martin

Dept. Math., Cox Western Reserve Univ.

Cleveland, Ohio 44106

\section{Abstract}

This note is concerned with the twin questions of how to recognize and define the concept of special structure for linear dynamical systems in a state-space basis independent way and how to exploit the fact that there is special structure to diminish to computational load of various solution procedures.

\section{INTRODUCTION.}

Many natural (and engineering) systems have a good deal of special structure (at least) intuitively. These can arise e.g. because the system is made of (many) components of only a few types, because there is a certain amount of decentralization, because there is an hierarchical command structure, because there is a certain inherent symmetry in the physics of the situation (Lagrangian and Hamiltonian systems), or because of a multitude of other possible reasons $[1,5,7]$.

This note is concerned with recognizing and exploiting special structure and with the topic of answering (solving) various system and control problems such as stabilization by feedback and DDP, ... in such a way that the special structure is preserved.

The tool is the symmetry algebra of a linear system.

This note only gives a short outline of the matrial presented in [5]. Related material is contained in[2] and [4].

\section{HOW TO RECOGNIZE SPECIAL STRUCTURE}

Let as consider a few examples.

\subsection{Twin lift helicopters.}

$$
\left[\begin{array}{l}
\dot{x}_{1} \\
\dot{x}_{2}
\end{array}\right]=\left(\begin{array}{cc}
A & H \\
-H & A
\end{array}\right]\left(\begin{array}{l}
x_{1} \\
x_{2}
\end{array}\right]+\left(\begin{array}{ll}
B & 0 \\
0 & B
\end{array}\right)\left(\begin{array}{l}
u_{1} \\
u_{2}
\end{array}\right)
$$

The $H$ represents the interaction of the two identical coupled helicopters on each other. For various practical reasons (cf. [5]) one is interested in finding out whether this system is state feedback stabilizable by means of a static feedback of the from $\left[\begin{array}{ll}k & 0 \\ 0 & k\end{array}\right]$. The (obvious) assumptions are that the total system is controllable and that the individual helicopters are controllable.

Intuitively at least this system definitely has special structure and feedback laws of the kind desired will preserve this special structure.

2.2. Example. consider the system

$$
\dot{x}=\left(\begin{array}{cc}
-6 & -3 \\
-13 & 10
\end{array}\right) x+\left(\begin{array}{ll}
1 & -2 \\
2 & -3
\end{array}\right) u
$$


Does this system have special structure? At first sight, no! However it is a base change transform of 2.1 and thus should have special structure

2.3. Example. Consider the system

$$
\dot{x}=\left[\begin{array}{lll}
\star & \star & 0 \\
\star & \star & \star \\
\star & \star & \star
\end{array}\right) x+\left[\begin{array}{ll}
\star & \star \\
\star & \star \\
\star & \star
\end{array}\right) u
$$

This one has no special structure. By suitable base changes any system can be brought into this form

2.4. Example. Consider a system of the form

$$
\dot{x}=\left(\begin{array}{cc}
A_{1} & 0 \\
A_{12} & A_{2}
\end{array}\right) x+\left(\begin{array}{c}
B_{1} \\
0
\end{array}\right) u
$$

This one should probably be considered to have special structure. But this is not reflected in the symmetry algebra of this class of system, cf. below, and thus this concept does certainly not capture all of the idea of special structure.

\section{THE SYMMETRY ALGEBRA OF A CLASS OF SYSTEMS.}

Let $\mathbf{C}$ be a class of systems. E.g. all systems of the form

$$
\dot{x}=F x+G u, F=\left(\begin{array}{cc}
A & H \\
-H & A
\end{array}\right), G=\left(\begin{array}{ll}
B & 0 \\
0 & B
\end{array}\right)
$$

Then the symmetry algebra of $\mathbf{C}$ is defined by

$$
R(\mathbf{C})=\left\{(S, T) \in M_{n}(\boldsymbol{R}) \times M_{m}(\boldsymbol{R}): S F=F S, S G=G T \text { for all }(F, G) \in \mathbf{C}\right\}
$$

$R(C)$ is an associative algebra. (Here $M_{n}(\boldsymbol{R})$ is the algebra of $n \times n$ matrices.)

Note that $\boldsymbol{R}^{n}$ is an $M_{n}(\boldsymbol{R})$-module and $\boldsymbol{R}^{m}$ an $M_{m}(\boldsymbol{R})$-module so that the state space $\boldsymbol{R}^{n}$ and the input space $\boldsymbol{R}^{\boldsymbol{m}}$ of a system in class $\mathbf{C}$ interit an $R(\mathbf{C})$-module structure. And then (3.1) says that $F$ is an $R(\mathbf{C})$-module endomorphism and $G$ and $R(\mathbf{C})$-module homomorphism. Thus $\Sigma=(F, G)$ becomes a (non free as a rule) system over the associative ring $R(\mathrm{C})$.

\section{EXAMPLES OF SYMMETRY ALGEBRAS.}

Consider first example 2.1. A basis for the symmetry algebra is formed by the pairs of matrices

$$
\left[\left(\begin{array}{cc}
I_{n} & 0 \\
0 & I_{n}
\end{array}\right],\left[\begin{array}{cc}
I_{m} & 0 \\
0 & I_{m}
\end{array}\right]\right),\left(\left[\begin{array}{cc}
0 & I_{n} \\
-I_{n} & 0
\end{array}\right],\left[\begin{array}{cc}
0 & I_{m} \\
-I_{n} & 0
\end{array}\right]\right)
$$

and the symmetry algebra is isomorphic to $\boldsymbol{R}[i] /\left(i^{2}=-1\right)=q$. An obvious fact.

4.1. Examples. Three units identically interconnected

$$
F=\left(\begin{array}{lll}
A & H & H \\
H & A & H \\
H & H & H
\end{array}\right), G=\left(\begin{array}{lll}
B & 0 & 0 \\
0 & B & 0 \\
0 & 0 & B
\end{array}\right)
$$

The symmetry algebra becomes the group algebra $\boldsymbol{R}\left[S_{3}\right]$, where $S_{3}$ is the symmetric group on 3 letters. This example obviously generalizes to $r$ identically interconnected units with symmetry algebra $R\left[S_{r}\right]$.

4.2. Example. Circulant systems [1].

$$
F=\left(\begin{array}{llll}
A_{1} & A_{2} & \ldots & A_{r} \\
A_{2} & A_{3} & \cdots & A_{1} \\
& & & \\
A_{r} & A_{1} & \cdots & A_{r-1}
\end{array}\right), G=\left(\begin{array}{cccc}
B_{1} & B_{2} & \ldots & B_{r} \\
B_{2} & B_{3} & \cdots & B_{1} \\
B_{1} & B_{1} & \cdots & B_{r-1}
\end{array}\right)
$$


The symmetry algebra becomes $R$ (circulant of order $r$ ) $=\mathbb{R}[\mathbb{Z} /(r)]=\mathbb{R}[X] /\left(X^{n}=1\right.$ ).

There are also examples with less familiar symmetry algebras as e.g. the 4-dimensional vectorspace $\boldsymbol{R} a \oplus \boldsymbol{R} b \oplus \boldsymbol{R} c \oplus \boldsymbol{R} 1$ with the multiplication rules $a^{2}=1, a b=b, b a=-b, a c=c a=c, b^{2}=b c=c b=c^{2}=0$, or the quaternion algebra $\boldsymbol{H}=\boldsymbol{R} \oplus \boldsymbol{R} i \oplus \boldsymbol{R} j \oplus \boldsymbol{R} k, i^{2}=j^{2}=k^{2}=-1, i j=k, j k=i, k i=j, j i=k, k j=-i, i k=-j$. In fact

4.3. Theorem. Let $R$ be a finite dimensional associative algebra over $R$. Then there exists a class of systems with symmetry algebra $R$.

\section{DEFINITION OF SPECIAL STRUCTURE.}

For each system $\Sigma=(F, G)$ let $R(\Sigma)$ be its symmetry algebra. Let $R$ be an associative algebra over $\boldsymbol{R}$. A system with special structure $R$ is now a system $(F, G)$ together with an injective ring homomorphism $R \rightarrow R(\Sigma)$. This notion is base-change invariant (but a base change does change the $R(\Sigma)$ as a subalgebra of $M_{n}(\boldsymbol{R}) \times M_{m}(\boldsymbol{R})$ (to an isomorphic one) and hence changes the embedding $R \rightarrow R(\Sigma)$ ).

\section{INTERMEZZO: SEMI-SIMPLE ALGEBRAS.}

A finite dimensional $\boldsymbol{R}$-algebra $R$ is said to be semi-simple if every submodule of an $R$-module has a complementary submodule. Thus every $R$-module breaks up as a direct sum of irreducible submodules. (A module $V$ is irreducible if every submodule of $V$ is zero or equal to $V$.)

Examples of semi-simple $\boldsymbol{R}$-algebras are $\mathcal{C} ; \boldsymbol{H}: \boldsymbol{R}[G], G$ a finite group $M_{n}(\boldsymbol{R})$.

Schur Lemma. Let $V, V^{\prime}$ be irreducible modules over $R$. Then $\operatorname{End}_{R}\left(V, V^{\prime}\right)=0$ unless $V$ and $V^{\prime}$ are isomorphic, and $\operatorname{End}_{R}(V)=\boldsymbol{R}, \boldsymbol{C}$, or $\boldsymbol{H}$.

\section{SYSTEMS WITH SEMI-SIMPLE SPECIAL STRUCTURE.}

Let $R$ be a semi-simple algebra and consider a class of systems with special structure $R$.

Theorem Scheme. The theory of systems with semi-simple special structure $R$ decomposes into the direct sum of the theories of ordinary systems over $\boldsymbol{R}, \boldsymbol{C}$ and $\boldsymbol{H}$.

This follows immediately from the decomposition of the state space and input space into a direct sum of irreducible $R$-modules and the Schur lemma. For some of the implications of this theorem scheme cf. [5]. E.g. the results of [1] follow immediately. A begining of the theory of systems over $\boldsymbol{H}$ has been made in [4].

\section{EXTRA SPECIAL STRUCTURE.}

The special structure preserving feedback laws for example 2.1 are feedback matrices of the form $\left(\begin{array}{cc}k_{1} & k_{2} \\ -k_{2} & k_{1}\end{array}\right)$ and this is not yet the form we are looking for. This, however, can also be formulated in terms of symmetry ideas. Roughly, extra special structure consists of two symmetry algebras $R \subset R^{\prime}$ such that $F$ is an $R$-module endomorphism and $G$ and $R^{\prime}$-module homomorphism. The feedback law is then required to be also an $R^{\prime}$ module homomorphism.

Output feedback can also be formulated in these terms 


\section{ON THE TWIN LIFT PROBLEM.}

Let us conclude with a few remarks on the problem of example 2.1 .

(i) For 2-dimensional helicopters (i.e. the $A$ is a $2 \times 2$ matrix) the desired stabilizing feedback always exists

(ii) There is a Heyman's lemma so that it suffices to solve the problem for the single input case

(iii) At the time of writing it seems to us not unlikely that there exist examples such that there exists a number $t$ such that if a feedback law $k=\left(k_{1}, \ldots, k_{m}\right)$ is such that ||$k|| \geqslant t$ then the matrix

$$
\left[\begin{array}{cc}
A & H \\
-H & A
\end{array}\right]+\left[\begin{array}{ll}
B & 0 \\
0 & B
\end{array}\right]\left[\begin{array}{ll}
k & 0 \\
0 & k
\end{array}\right)
$$

is unstable.

\section{FINAL REMARK.}

So far only the case of semi-simple special structure has been exploited somewhat. It seems to us that e.g. the presence of a symmetry algebra with a nilpotent ideal will also lead to interesting system theoretic consequences (e.g. of the cascade decomposition sort). In general there are probably many results from representation theory which can be exploited.

\section{References}

1 R.W. Brockett, J.L. Willems, Discretized partial differential equations: examples of control systems defined on modules, Automatica 10 (1974), 507-515.

2 R. Grossman, C.F. Martin, Approximation and control of symmetric systems on the circle, Preprint, 1983.

3 C.W. Curtis, I. Reiner, Representation theory of finite groups and associative algebras, Interscience, 1962.

4 M. Hazewinkel, J.H. Lewis, C.F. Martin, Symmetric systems with semi-simple structure algebra: the quaternionic case, Systems and Control Letters 3 (1983), to appear. (Report BW 185/83, Math. Centre, Amsterdam).

5 M. Hazewinkel, C.F. Martin, On decentralization, symmetry and specialization in linear systems, Submitted SIAM J. Control and Opt., Report 8201, Econometric Inst., Erasmus Univ. Rotterdam.

6 N. Jacobson, The theory of rings, Amer. Math. Soc. 1943.

7 T.J. Tarn, G. Huang, J.W. Clark, Modeling of quantum mechanical control systems, Math. Modeling 1 (1980), 109-121. 